\title{
Uso del plerixafor en pacientes pobres movilizadores para trasplante autólogo
}

\author{
Plerixafor use in poor mobilizer patients for autologous transplant
}

\author{
- Juan Manuel Herrera', Jorge E. Duque², Nohra Bolívar³, Marcela Urrego ${ }^{4}$, Rigoberto Gómez ${ }^{5}$, Álvaro J. Guerrero ${ }^{6}$, \\ Rocío Salcedo7, Johana Buitrago ${ }^{8}$ \\ Coordinador trasplante, Centro Médico Imbanaco. \\ Médico hematooncólogo, Centro Médico Imbanaco. \\ Servicio de transfusión, Centro Médico Imbanaco. \\ Médica hematooncologa, Centro Médico Imbanaco. \\ Médico hematologo clinico, Centro Medico Imbanaco. \\ Enferma \\ ${ }_{8}$ Coordinadora de Enfermería, Centro Médico Imbanaco.
}

\section{Resumen}

El trasplante autólogo es el tratamiento estándar para pacientes con mieloma múltiple (MM) o linfoma no Hodgkin de grados intermedio o alto (LNH) con recaídas quimiosensibles, permitiendo rescatar los pacientes después de recibir quimioterapia a dosis ablativas. En Colombia, en el año 2012 se realizó 1 trasplante por 100 mil habitantes (435 pacientes entre autólogos y alogénicos), siendo un país con bajo promedio de trasplantes per capita.

Objetivo: describir las características clínicas y desenlaces de pacientes pobres movilizadores sometidos a trasplante en el Centro Médico Imbanaco de Cali y que recibieron plerixafor para un segundo intento de movilización o para realizar trasplante alogénico.

Materiales y métodos: estudio descriptivo retrospectivo basado en los registros médicos del Centro Médico Imbanaco de Cali durante el período 2004 a 2015. Se tuvieron en cuenta edad, sexo, número de aféresis, demora, recolección y recuperación.

Resultados: se efectuaron 207 trasplantes autólogos, 196 en adultos y 11 en niños. Dentro de los adultos, 5 pacientes $(2,55 \%)$ fueron catalogados como pobres movilizadores, y de estos, 4 fueron removilizados con plerixafor más G-CSF y se hicieron 11 aféresis en los cuatro pacientes. Todos los pacientes pudieron ser llevados a trasplante autólogo recibiendo acondicionamientos convencionales, BEAM para linfomas y melfalán para el paciente con mieloma múltiple. Todos los pacientes salieron de aplasia en promedio a los 14,25 días (rango 13 a 15).

Conclusiones: el plerixafor permite movilización y recolección de progenitores hematopoyéticos en pacientes 'pobres movilizadores', pudiendo trasplantarse. La demora en trámites administrativos pone en grave peligro la vida de los pacientes.

Palabras clave: trasplantes, enfermedades hematológicas, linfoma no Hodgkin, mieloma múltiple, movilización de célula madre hematopoyética.

\begin{abstract}
Autologous transplantation is the standard treatment for patients with multiple myeloma or intermediate or high grade nonHodgkin's lymphoma with chemosensitive relapses, which allows patients to recover after receiving chemotherapy at ablative doses. In Colombia, in 2012, 1 per 100 million inhabitants were made (435 patients among autologous and aloal), being a country with low average transplants per person.

Objective: To describe the clinical characteristics and the triggers of poor mobilizing patients in a transplant at the Imbanaco Medical Center in Cali and in which paragraphs were received for a second mobilization attempt or to perform the allogeneic transplant.

Materials and methods: A retrospective descriptive study based on the medical records of the Imbanaco Medical Center of Cali, from 2004 to 2015. Age, sex, number of apheresis, delay, collection and recovery were taken into account.

Results: 207 autologous transplants were performed, 196 in adults and 11 in children. Within adults, 5 patients (2.55\%) were classified as poor mobilizers, and of these, 4 were remobilized with plerixa for more G-CSF and 11 aphereses were made in the 4 patients. All the patients were taken to an autologous transplant that received conventional conditioning, BEAM for lymphomas and melfalan for the patient with multiple myeloma. All the patients left aplasia, on average at 14.25 days (range 13 to 15 ).
\end{abstract}

Recibido: 10 de octubre de 2016: aceptado: 25 de junio de 2018 
Conclusions: plerixafor allows mobilization and collection of hematopoietic progenitors in patients 'poor mobilizers', and can be transplanted. The delay in administrative procedures endangers the lives of patients.

Key words: Transplantation, autologous, plerixafor, hematological diseases.

\section{Introducción}

El trasplante de médula ósea es una opción terapéutica con intención curativa en enfermedades benignas y malignas; el trasplante puede ser de médula ósea propiamente dicha o de células madre de sangre periférica o hasta de sangre de cordón umbilical. También se divide en trasplante autólogo si es el propio paciente el que hace la donación; o alogénico, pudiendo ser este último singénico en caso de gemelo idéntico y emparentado o no emparentado, ya sea de donante HLA idéntico o compatible (vg 1 mismatch) o haploidéntico.

El trasplante autólogo es el tratamiento estándar para pacientes con mieloma múltiple (MM) o linfoma no Hodgkin de grados intermedio o alto $(\mathrm{LNH})$ con recaídas quimiosensibles, ya que permite rescatar los pacientes después de recibir quimioterapia a dosis ablativas'.

El éxito del trasplante autólogo depende de muchos factores, siendo uno de los más importantes la dosis de células madre reinfundidas ${ }^{2,3}$. Dosis más altas son asociadas a un prendimiento más rápido del injerto, siendo definido como recuento de plaquetas mayor de 20 mil por tres días consecutivos, en ausencia de transfusión y un recuento absoluto de neutrófilos (RAN) mayor de 5004. Existen además factores que afectan la recolección de progenitores, como son la edad, el sexo, la exposición previa a quimio o radioterapia, trasplantes previos o compromiso de la médula ósea por la enfermedad de base. El fracaso de la primera movilización lleva a costosos intentos de removilizar, que pueden llegar a impedir la realización del trasplante ${ }^{5}$.

Durante el año 2013 se registraron más de 320 mil trasplantes ${ }^{6}$ en el Registro Internacional de Trasplante de Médula Ósea (CIBMTR, por su sigla en inglés), mientras en Colombia en el año 2012 se hicieron 435 trasplantes o procedimientos entre autólogos y alogénicos, lo que significa o se traduce en un 1 trasplante por cada 100 mil habitantes aproximadamente, siendo un país con bajo promedio de trasplantes per capita7.

\section{Técnicas de movilización}

El objetivo de las células madre recolectadas es repoblar la médula ósea después de la quimioterapia ablativa. Aunque en todo momento hay en la sangre periférica una pequeña cantidad de células madre, es necesario hacer la movilización de estas para poder colectar el número suficiente de ellas por aféresis y poder llevar los pacientes a trasplante. La sangre periférica es superior a la médula ósea como fuente de células madre, pues su recolección es más sencilla, hay menor morbilidad y el tiempo para el prendimiento es más corto ${ }^{8}$. El objetivo de la recolección es obtener un mínimo de $2 \times 10^{6}$ células a un máximo de $4 \times 10^{6}$.

\section{Movilización inducida por citoquinas}

Los factores de crecimiento hematopoyético GMCSF (sargramostim), G-CSF (filgrastim) y pegfilgrastim (filgrastim pegilado) pueden ser usados para la movilización de progenitores hematopoyéticos. El GM-CSF es poco usado por tener más efectos secundarios y obtener bajo número de progenitores ${ }^{9}$.

Los mecanismos biológicos a través de los cuales las citoquinas inducen la movilización de progenitores son complejos y no están completamente entendidos. Sin embargo, es conocido que las células madre tienen un pico en sangre periférica al quinto día después de la aplicación de G-CSF ${ }^{10}$.

\section{Movilización con G-CSF}

La aplicación de G-CSF induce cambios funcionales dentro del microambiente de la médula ósea y las enzimas liberadas por las células mieloides rompen las moléculas de adhesión y las interacciones entre citoquinas, sus receptores y la matrix extracelular. En presencia de GCS-F, el número de progenitores comprometidos al linaje mieloide se aumenta, causando liberación y activación de la catepsina $\mathrm{G}$ y de la elastasa de los neutrófilos, dentro del líquido extracelular de la médula ósea. Esto produce ruptura de las moléculas de adhesión, como c-kit, molécula -I, CXCR4 y SDF-1"11.

El G-CSF es el factor más usado para movilización de progenitores. Se utiliza a dosis de $10 \mathrm{mcg} / \mathrm{kg} / \mathrm{día}$, repartido en dos dosis al día, se empieza por lo menos cuatro días antes de la primera aféresis y se coloca hasta la última recolección. La movilización con G-CSF es bien tolerada y los eventos adversos más frecuentes son cefalea, dolores óseos, baja de número de plaquetas 
y anemia. Hay otros más infrecuentes, como fatiga, dolor muscular, náusea y vómito, y aún más raros, como infarto al miocardio o isquemia cerebral en los pacientes de alto riesgo de sufrirlo, ruptura esplénica o crisis falciforme en pacientes con esta enfermedad ${ }^{10}$.

La eficacia del G-CSF para la movilización en trasplante autólogo ya ha sido establecida en estudios fase III. Schimtz y colaboradores lo usaron en 58 pacientes con linfoma Hodgkin y no Hodgkin, utilizándolo seis días y obteniendo un promedio de 2,86 × $10^{6}$ células CD34(+)/ $/ \mathrm{kg}^{11}$. Además, al comparar la recuperación hematológica de leucocitos y plaquetas con sangre periférica o con médula ósea, fue mucho más corta con la primera ${ }^{12}$. Existen pacientes malos movilizadores, que no logran recolectar un mínimo de $2 \times 10^{6}$ células CD34(+)/kg, lo cual es más frecuente cuando se usa la movilización solo con G-CSF que cuando se combina con quimioterapia. Estos malos movilizadores corresponden del $0 \%$ al $23 \%$ de los $\operatorname{casos}^{13}$. Se han identificado factores asociados a la mala movilización, como el compromiso de la médula ósea por su enfermedad de base, el número de ciclos de quimioterapia recibidos y el uso de fludarabina o lenalidomida.

\section{G-CSF con quimioterapia}

Antes de la aparición de las citoquinas, la movilización se realizaba solamente con quimioterapia; después de su descubrimiento, se notó que su uso diario luego de la quimioterapia aumentaba el número de progenitores ${ }^{14}$. El mecanismo por el cual la combinación de quimioterapia y citoquinas incrementa el número de células madre no ha sido completamente entendido. El empleo de agentes quimioterapéuticos a dosis que induzcan aplasia aumentan la recuperación hematológica. El pico es variable y sucede entre 10 a 18 días después de su aplicación, y generalmente coincide con la elevación del número de neutrófilos.

Hay una gran variedad de agentes quimioterapéuticos que son usados para la movilización de progenitores hematopoyéticos, siendo la ciclofosfamida el más ampliamente utilizado, o, en ocasiones, se aprovechan los esquemas de quimioterapia de rescate para la movilización. La quimio/movilización es ampliamente usada, pues se incrementa el número de CD34(+) comparada con la movilización con solo G-CSF, necesitándose entonces menos sesiones de aféresis ${ }^{15}$. Aunque los regímenes de movilización que incluyen quimioterapia y G-CSF reducen el riesgo de contaminación por células tumorales del producto recolectado, hay mucho debate sobre si esto concede un beneficio en la sobrevida de los pacientes.

La adición de quimioterapia a los esquemas de movilización no está exenta de riesgos, como son infección, uso de antibióticos, hospitalizaciones, transfusiones y mayor costo, aunque la mortalidad es relativamente rara $^{16}$. Además, hay un mayor número de complicaciones entre más alta sea la dosis de quimioterapia que se use. Adicionalmente, los picos de CD34(+) pueden tener variaciones entre los pacientes, lo que hace difícil programar la fecha de la recolección, y es necesaria la medición de CD34(+) en sangre periférica varios días para poder hacer la cosecha. Aquellos pacientes en los que la utilización de quimio y G-CSF falla para obtener un número adecuado de progenitores son los llamados malos movilizadores y son susceptibles del uso de plerixafor.

\section{Pegfilgrastim}

Es el G-CSF pegilado, con una vida media de 33 horas, en comparación con las 6 horas del G-CSF corriente. Su rata lenta de eliminación renal permite tener niveles por tiempo prolongado hasta la salida de la aplasia ${ }^{17}$. Hay ya experiencias, con la aplicación de pegfilgrastim a las 24 horas de la aplicación de la quimioterapia, teniendo un pico de CDE34(+) hacia los 13 días (rango 11 a 22) ${ }^{18}$. El pegfilgrastim es bien tolerado y su perfil de seguridad es similar al del G-CSF; se ha además informado dolor torácico, náusea y un caso de ruptura esplénica.

\section{Movilización inducida con plerixafor}

El plerixafor (AMD 3100) es un antagonista reversible y selectivo de CXCRC4, alterando su interacción con SDF-1, librando así las células madre a la circulación ${ }^{19}$. En pacientes en quienes la movilización con G-CSF o quimio y G-CSF falla, la utilización de G-CSF más plerixafor puede aumentar entre 5 a 10 veces el número de células CD34(+ $)^{20}$. En estudios randomizados, los pacientes recibieron G-CSF a dosis de $10 \mathrm{mcg} / \mathrm{kg}$ día por cuatro días en la tarde del día 4, una dosis de 240 $\mathrm{mcg} / \mathrm{kg}$ sc de plerixafor, hasta hacer cuatro aféresis o hasta un máximo de $5 \times 10^{6}$ células CD34(+). En esta forma, se pueden recolectar un mínimo de $2 \times 10^{6}$ células en más del $60 \%$ de los pacientes que fallaron por otros medios 


\section{Hasta cuánto recolectar}

Se considera suficiente una recolección de $2 \mathrm{x}$ $10^{6}$ células $/ \mathrm{kg} \mathrm{y}$, aunque menores cantidades se han usado, esto conlleva mayor tiempo de aplasia tanto de neutrófilos como de plaquetas. Varios estudios han demostrado que el número óptimo podría ser mayor de $5 \times 10^{6}$ células, mostrando una más rápida y robusta recuperación hematológica y además una mayor sobrevida total21.

\section{Materiales y métodos}

Se hizo revisión de las historias clínicas de todos los pacientes de todas las edades sometidos a trasplante en el Centro Médico Imbanaco de Cali y se determinó cuáles pacientes habían sido pobres movilizadores y si se había usado el plerixafor para hacer un segundo intento de movilización o para ser llevado a trasplante alogénico. Una vez identificados, se establecieron tiempos para poder hacer el segundo intento de recolección y además salida de aplasia terapéutica, dada por un recuento de neutrófilos $<500$ por tres días consecutivos.

\section{Resultados}

De 2004 a 2015 se han realizado 207 trasplantes autólogos en el Centro Médico Imbanaco de Cali. De estos, 196 son adultos y 11 pediátricos. Dentro de los adultos, 5 pacientes fueron catalogados como pobres movilizadores, lo que corresponde al $2,55 \%$, y de estos, 4 fueron removilizados con plerixafor y 1 paciente con linfoma fue llevado directamente a trasplante haploidéntico. De los otros, 3 tenían diagnóstico de linfoma no Hodgkin y 1 de mieloma múltiple.

Los cuatro pacientes recibieron la combinación de plerixafor más G-CSF, como está descrito, y se hicieron 11 aféresis en los cuatro pacientes, obteniendo 3,91 x $10^{6}$ células CD34(+) (rango de 2,33 a 5,93), y así todos los pacientes pudieron ser llevados a trasplante autólogo recibiendo acondicionamientos convencionales, BEAM para los linfomas y melfalán para el paciente con mieloma múltiple. Todos los pacientes salieron de aplasia en promedio a los 14,25 días (rango 13 a 15) (ver tabla 1).

Los cuatro pacientes fueron solo movilizados con G-CSF inicialmente y un paciente (número 2) al hacer la solicitud del Plerixafor a la aseguradora, esta exigió que fuera reintentada la movilización con el uso previo de Ciclofosfamida, lo cual se hizo sin resultados positivos. Mientras se llenaban los requisitos de autorizaciones ante su aseguradora y el Invima, pasaron 79,2 días (rango de 49 a 160 días).

\section{Discusión}

El trasplante autólogo es un procedimiento terapéutico que puede mejorar la calidad de vida de los pacientes o llevar hasta la curación. Como generalmente los pacientes han recibido tratamientos previos, en algunos la obtención de células CD34(+) suficientes para llevar a trasplante puede ser difícil o imposible. Estos pacientes podrían perder la oportunidad de ser llevados a trasplante. En nuestro Centro utilizamos como umbral ideal para llevar a trasplante 3,5 × $10^{6}$ células CD34(+) para autólogo y como mínimo $2 \times 10^{6}$. Usamos un máximo de cinco recolecciones para obtener este número. Esto explica por qué nuestro número de pacientes pobres movilizadores es más bien bajo al compararlo con la recomendación actual, que es de $5 \times 10^{6}$ células como lo ideal, aunque mantiene el mínimo de $2 \times 10^{6}$.

Nuestra experiencia es muy pequeña, pero todos nuestros pacientes movilizaron un adecuado número de células CD34(+) con la utilización del plerixafor y pudieron así ser llevados a trasplante; ninguno falleció y todos tuvieron recuperación de neutrófilos y plaquetas (este último dato no mostrado) en forma adecuada.

Lo que sí fue preocupante fue la demora de 79,2 días entre la primera aféresis y la movilización exitosa con plerixafor. Esto está dado por el alto costo del medicamento, por lo que requiere trámites adicionales con las aseguradoras, además de que, cuando tratamos estos pacientes, el plerixafor no tenía registro del Instituto

Tabla 1. Características de los pacientes

\begin{tabular}{|c|c|c|c|c|c|c|}
\hline Paciente & Edad (años) & Sexo & Aféresis (\#) & Demora (días) & Recolección (CD34) & Recuperación (días) \\
\hline 1 & 45 & M & 3 & 55 & 2,33 & 14 \\
\hline 2 & 59 & $\mathrm{~F}$ & 3 & 55 & 3,42 & 15 \\
\hline 3 & 62 & $\mathrm{~F}$ & 2 & 49 & 3,88 & 14 \\
\hline 4 & 57 & M & 3 & 160 & 5,93 & 14 \\
\hline Total & 54,7 & & 3,75 & 79,2 & 3,87 & 14,25 \\
\hline
\end{tabular}


Nacional de Vigilancia de Medicamentos y Alimentos (Invima) y su importación era dispendiosa.

Nuestro sistema de salud hace muy difícil programar la recolección de células CD34(+) después de un ciclo de quimioterapia de rescate, lo cual sería lo ideal, por lo que nuestro método de movilización preferido es el uso de G-CSF solo y en aquellos pacientes con antecedentes ( $v$. g., segundos trasplantes, uso de previo de lenalidomida por más de cuatro ciclos o fludarabina) lo hacemos generalmente con quimioterapia y G-CSF.

\section{Conclusiones}

El plerixafor permite la movilización y recolección de progenitores hematopoyéticos en pacientes catalogados como 'pobres movilizadores', pudiendo trasplantarse. La demora para cumplir trámites administrativos pone en grave peligro la vida de los pacientes, por lo que es necesario que los centros de trasplante diseñen estrategias para acortar estos tiempos.

\section{Referencias}

1. Philip T, Guglielmi C, Hagenbeek A, Somers R, Van der Lelie $H$, Bron $D$, et al. Autologous bone marrow transplantation as compared with salvage chemotherapy in relapses of chemotherapy-sensitive non-Hodgkin's lymphoma. N Engl J Med. 1995;333(23):1540-5.

2. Child JA, Morgan GJ, Davies FE, Owen RG, Bell SE, Hawkins K, et al. High-dose chemotherapy with hematopoietic stem-cell rescue for multiple myeloma. N Engl J Med. 2003;348(19):1875-83.

3. Jillella AP, Ustun C. What is the optimum number of CD34+ peripheral blood stem cells for an autologous transplant? Stem Cells Dev. 2004;13(6):598-606.

4. Tricot $G$, Jagannath $S$, Vesole D, Nelson J, Tindle S, Miller L, et al. Peripheral blood stem cell transplants for multiple myeloma: identification of favorable variables for rapid engraftment in 225 patients. Blood. 1995;85(2):588-96

5. Goterris R, Hernández-Boluda JC, Teruel A, Gómez C, Lis MJ, Terol MJ, et al. Impact of different strategies of second-line stem cell harvest on the outcome of autologous transplantation in poor peripheral blood stem cell mobilizers. Bone Marrow Transpl. 2005;36(10):847-53

6. Pasquini M, Wang Z. Current use and outcome of hematopoietic stem cell transplantation: CIBMTR summary slides [internet]. 2013. Disponible en: http://www.cibmtr.org2013.

7. Instituto Nacional de Salud (INS), Dirección Redes en Salud Pública, Subdirección Red Nacional de Trasplantes y Bancos de Sangre, Coordinación Nacional Red Donación y Trasplante. Informe anual red de donación y trasplantes. 2013.

8. Schmitz $N$, Linch $D$, Dreger $P$, Goldstone $A$, Boogaerts $M$, Ferrant A. Randomised trial of filgrastim mobilized peripheral blood progenitor cell transplantation versus autologous bone-marrow transplantation in lymphoma patients. Lancet. 1996;347:353-7.

9. Gazitt Y. Comparison between granulocyte colony-stimulating factor and granulocyte-macrophage colony-stimulating factor in the mobilization of peripheral blood stem cells. Curr Opin Hematol. 2002;9(3):190-8.

10. Neupogen (filgrastim) [package insert]. Thousand Oaks, CA: Amgen Inc.

11. Levesque JP, Liu F, Simmons PJ, Betsuyaku T, Senior RM, Pham $C$, et al. Characterization of hematopoietic progenitor mobilization in protease-deficient mice. Blood. 2004;104(1):65-72.
12. Schmitz N, Linch DC, Dreger $P$, Goldstone $A H$, Boogaerts $M A$, Ferrant $A$, et al. Randomised trial of filgrastim-mobilised peripheral blood progenitor cell transplantation versus autologous bone-marrow transplantation in lymphoma patients. Lancet. 1996;347(8998):353-7.

13. Moskowitz CH, Glassman JR, Wuest D, Maslak P, Reich L, Gucciardo $A$, et al. Factors affecting mobilization of peripheral blood progenitor cells in patients with lymphoma. Clin Cancer Res. 1998;4(2):311-6.

14. Socinski MA, Cannistra SA, Elias A, Antman KH, Schnipper $L$, Griffin JD. Granulocyte-macrophage colony stimulating factor expands the circulating haematopoietic progenitor cell compartment in man. Lancet. 1988;1(8596):1194-8.

15. Ford C, Greenwood J, Anderson J, Snow G, Petersen FB. CD34+ cell adhesion molecule profiles differ between patients mobilized with granulocyte-colony-stimulating factor alone and chemotherapy followed by granulocyte-colony-stimulating factor. Transfusion. 2006;46(2):193-8.

16. Fitoussi O, Perreau V, Boiron JM, Bouzigon E, Cony Makhoul P, Pigneux $A$, et al. A comparison of toxicity following two different doses of cyclophosphamide for mobilization of peripheral blood progenitor cells in 116 multiple myeloma patients. Bone Marrow Transpl. 2001;27(8):837-42.

17. Neulasta (pegfilgrastim) [package insert]. Thousand Oaks, CA: Amgen Inc.; 2007

18. Fruehauf S, Klaus J, Huesing J, Veldwijk MR, Buss EC, Topaly J, et al. Efficient mobilization of peripheral blood stem cells following CAD chemotherapy and a single dose of pegylated G-CSF in patients with multiple myeloma. Bone Marrow Transpl. 2007;39(12):743-50.

19. Hatse S, Princen K, Bridger G, De Clercq E, Schols D. Chemokine receptor inhibition by AMD3100 is strictly confined to CXCR4. FEBS Lett. 2002;527(1-3):255-62.

20. Flomenberg N, Devine SM, Dipersio JF, Liesveld JL, McCarty JM, Rowley SD, et al. The use of AMD3100 plus G-CSF for autologous hematopoietic progenitor cell mobilization is superior to G-CSF alone. Blood. 2005;106(5):1867-74.

21. Stiff PJ, Micallef I, Nademanee AP, Stadtmauer EA, Maziarz RT, Bolwell BJ, et al. Transplanted CD34(+) cell dose is associated with long-term platelet count recovery following autologous peripheral blood stem cell transplant in patients with nonHodgkin lymphoma or multiple myeloma. Biol Blood Marrow Transpl. 2011;17(8):1146-53. 